\title{
Influences of sensor protective coating on laser energy calibration results
}

\author{
T. Oytun Kılınç ${ }^{1, *}, K$. Berk Sönmez ${ }^{1}, \dot{I}$. Ahmet Yüksel ${ }^{1}$, and Sinem Ön Aktan ${ }^{1}$ \\ ${ }^{1}$ Department of Laboratories and Calibration Management, Roketsan Missiles Industries Inc., 06780 \\ Ankara, Turkey
}

\begin{abstract}
Improving laser energy measurement is a challenging task. Pyroelectric crystalline energy sensors used in laser energy measurements have a working principle that absorbs the heat of incoming light. Therefore, it is very important to measure the heat transfer in the incoming laser beam with maximum efficiency. The condition of the black sensor coating used to maximize this efficiency plays a leading role in the measurements. By means of laser energy sensor calibration measuring setup, the energy of the pulsed laser source at a wavelength of $1064 \mathrm{~nm}$ and a frequency of $20 \mathrm{~Hz}$ was measured with damaged and undamaged black sensor coatings. The present study may provide useful guidance into the understanding of black coatings of the detectors to use in laser energy calibrations.
\end{abstract}

\section{Introduction}

Electromagnetic wave detection systems used in todays and future engineering solutions facilitate humanity's requirements in terms of minimum time and maximum efficiency. Intensive, coherent and monochromatic light beam in the detection system have a wide range of usage area includes civil and military applications. Not only these areas but also detection of radiation has an importance in many branches of science and technology. In older thermal radiation detectors, such as thermopiles and bolometers, this is regular application to increase the absorption of the receiver by using black coating [1]. Since electromagnetic radiation encompasses a wide spectrum, the detection devices and techniques depend on the wavelengths and energies involved. For this type of sensing systems must have a great importance about measurement accuracy. Therefore, calibration has an importance in sensing systems that respond quickly to a wide range electromagnetic spectrum.

Lasers are high intensity coherent sources of photons. It is possible to measure beam energy and power according to the heat transfer of the incoming laser beam is pulsed and continuous. Pyroelectric sensors produce a signal related to the amount of incident radiant energy. These types of sensors are commonly used for measuring the energy of laser pulses.

Pyroelectric thermal detectors are so distinctly from thermopiles and bolometers that it is unclear how similarly they will be affected by black coatings. An uncoated pyroelectric receiver is generally greater than $5 \mu \mathrm{m}$ thick and therefore has a much greater thermal

* Corresponding author: oytun.kilinc@roketsan.com.tr 
capacity per unit area than fast thermopile and bolometer receivers, typically metal foils of $0.1 \mu \mathrm{m}$ in thickness [1].

Although it is not the aim of this research to create either devices or standards for energy measurements, fruitful information modification may be useful for many instrument users.

Here we are investigating the effect of sensor coating on measurement results with using $1064 \mathrm{~nm}$ wavelength pulsed laser source without dealing with the energy meter. As shown in Fig. 1 a laser energy sensor which is manufactured by a dedicated well-known company with $153 \mathrm{~mm}$ diameter disk head and $95 \mathrm{~mm}$ diameter aperture with black coating were chosen for study on the basis of working principle, sensor structures, coating and uncoated performance and measurement uncertainty.

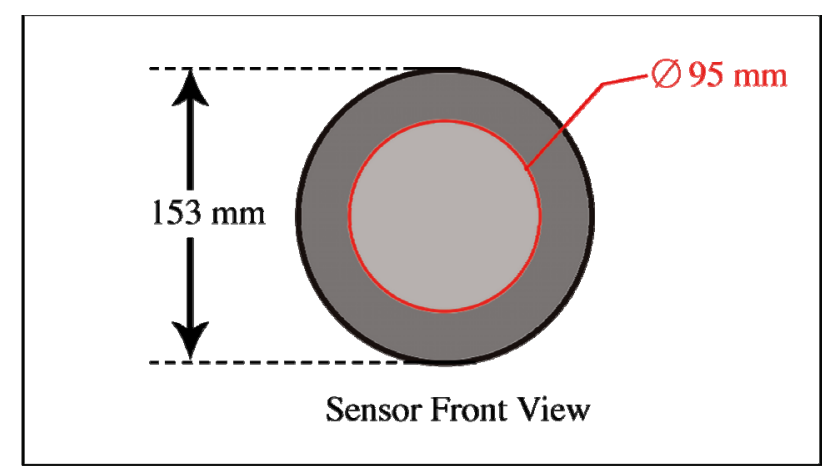

Fig. 1. Technical drawing of the pyroelectric sensor.

\section{Methodology}

In tests where energy is measured by laser pulse, incoming beam first encounters the first coating on the sensor. Then, the medium of the incoming laser beam changes for the first time at this layer which protects the detector. After this step laser energy is faced with pyroelectric material. Pyroelectric sensors generally measure pulsed energy at up to 25 $\mathrm{kHz}$. Pyroelectricity is one of the hidden electrical properties of solid materials. The theoretical treatment of pyroelectricity in terms of a change in net dipole moment has emerged in the modern age, although humanity is known as a phenomenon for more than 24 centuries. This has been observed in the tourmaline crystal, which is capable of attracting straws and wood fragments $[2,3]$.

Methodology was examined under three headings: working principle of the pyroelectric sensor, coating material on the detector and laser pulsed energy measurement method.

\subsection{Working principle}

Pyroelectric detectors have a pyroelectric crystal that generates an electric charge depending on to the heat absorbed. The response time of this sensor depends on the time which takes for the heat to enter the crystal and heat it up. This time is tens of $\mu$ s and so the metallic detectors can run at a high repetition rate. Clearly, Fig. 2 shows the energy of the incoming laser first encounters a black coating. This black coating is very successful for trapping heat. When the trapped heat interacts with the pyroelectric material, the electric charge from the electrodes is generated. Thus, the output power of the incoming laser energy can be read easily. 


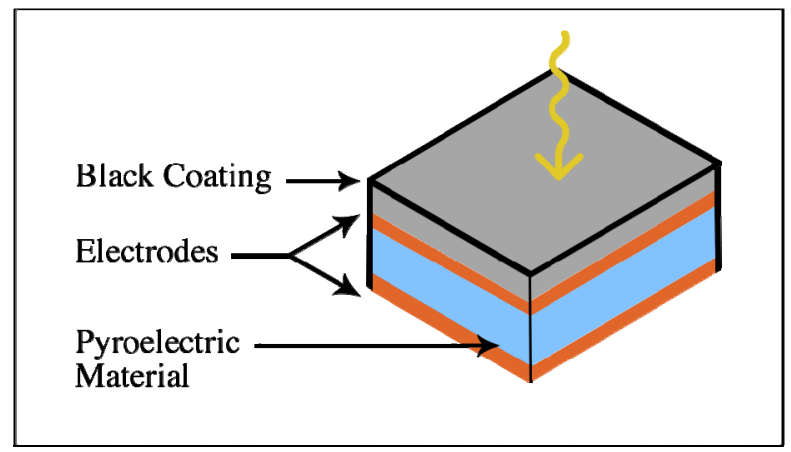

Fig. 2. Schematic view of the pyroelectric sensor.

In order to support the working principle, a scan with x-ray imaging support was performed to observe the internal structure of our pyroelectric sensor. When we look at the front view, side view and rear view of our sensor, it is observed that the pyroelectric material inside the sensor is similar to the battery (see Fig. 3).

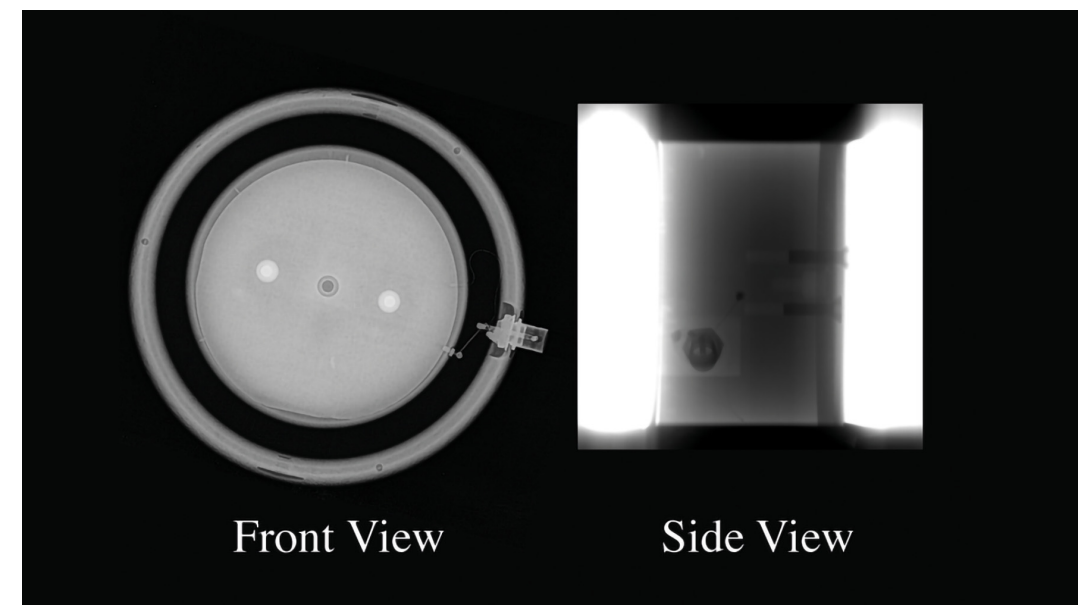

Fig. 3. X-Ray images of the pyroelectric sensor.

\subsection{Coating material}

A perfect coating for a detector must have a minimum reflectance independent of wavelength and angle properties. This has been particularly investigated using unique instruments. The incoming radiation is absorbed and the resulting temperature increase in the sensitive element often has an electrical effect like separating the charge in the pyroelectric detector. Pyroelectric sensors are usually used in a form that radiation absorption takes place in the pyroelectric crystal material or ceramic rather than at its surface. However, this limits the wavelength range in which they are useful. It is necessary to apply a black coating in order to extend the spectral range and providing an absorbing area of known spectral absorptance [4]. Preparation of detector black coatings seems like an art. When coatings are compared platinum black and the aluminium black have diffuse components of reflectance that are low throughout the middle infrared to far infrared. Nickel black is the diffuse material make an important contribution to the total reflectance [4]. Platinum black from finely divided metal coatings gives the best results and it has a 
consistently low reflectance speciality until $12 \mu \mathrm{m}$ wavelength. And also, nickel black is pretty good approximately up to $8 \mu \mathrm{m}$ wavelength. Finally, aluminium black is the least satisfactory material [4].

\subsection{Measurement method}

To use a laser energy sensor is a popular method to see the pulsed laser energy. When setup a measurement system, it is very important to know what to measure. In addition, it is always necessary to have a reference measuring instrument to ensure the accuracy and consistency of the results. To verify the operational principle of our measurement system, we have compared the system with reference energy detector. After this verification, in this setup, test sensor was replaced with reference detector. Measurements were taken between $30 \mathrm{~mJ}$ and $80 \mathrm{~mJ}$ with $1064 \mathrm{~nm}$ wavelength pulsed laser source. These measurements are carried out with a laser source, beam expander and a test detector. Optics such as polarizers, beam splitters or quarter wave-plates can be provided upon request.

While energy was measuring on the detector, beam expander must be used because test detector has a large aperture. This beam expander has to be matching the sensor area so homogeneous measurements can be taken. Otherwise, if small beam is used, measurement results can changeable on the aperture. We considered that there could be some performance problems about coating damage while using laser pulse energy without beam expander. Moreover, some damage problems on the coating could be seen because of high energy (see Fig 4).

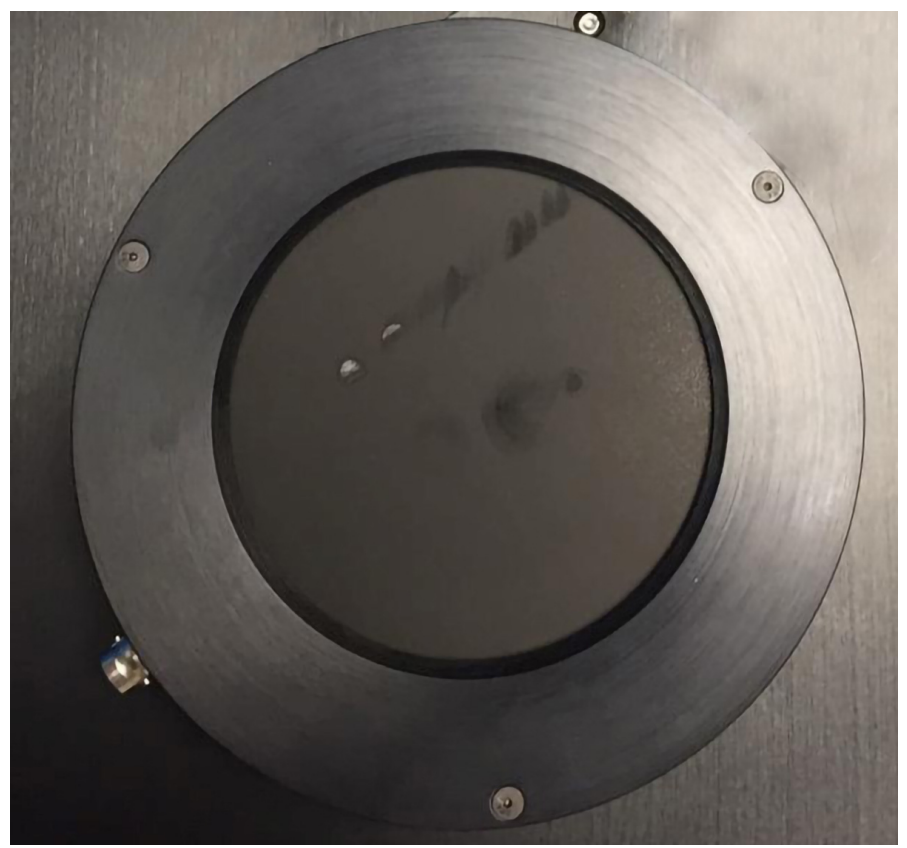

Fig. 4. Image of the coating damage sensor

Although the use of optics such as beam expander reduces the energy of the laser, each sensor has its own coating specific energy protection, and sometimes users may damage the detector's coatings during the measurement. Therefore, coating and uncoated surface of the detector performance must be examined. 


\section{Measurement performances}

The black coating of the test sensor was measured separately for defective and undamaged surfaces. All measurements were taken with the help of the reference sensor as the standard verification method of the test sensors. Energy measurement results were taken between 30 $\mathrm{mJ}$ and $80 \mathrm{~mJ}$ at $23 \pm 1{ }^{\circ} \mathrm{C}$ and $35 \% \mathrm{RH}$. And also, these measurements were noted by averaging 50 laser pulses with $1064 \mathrm{~nm}$ wavelength at $20 \mathrm{~Hz}$. Undamaged coating performances from these energy measurements shown in Table 1.

Table 1. Undamaged coating performance of the sensor

\begin{tabular}{|c|c|c|c|c|}
\hline \multicolumn{5}{|c|}{ Energy Measurements From Undamaged Coating } \\
\hline $71.3 \mathrm{~mJ}$ & $61 \mathrm{~mJ}$ & $49.9 \mathrm{~mJ}$ & $40.1 \mathrm{~mJ}$ & $33.5 \mathrm{~mJ}$ \\
\hline
\end{tabular}

While we were taking undamaged coating area measurement results from the test sensor, we excluded the damage coating areas. On the other hand, we examined damaged areas in three sections (see Fig. 5).

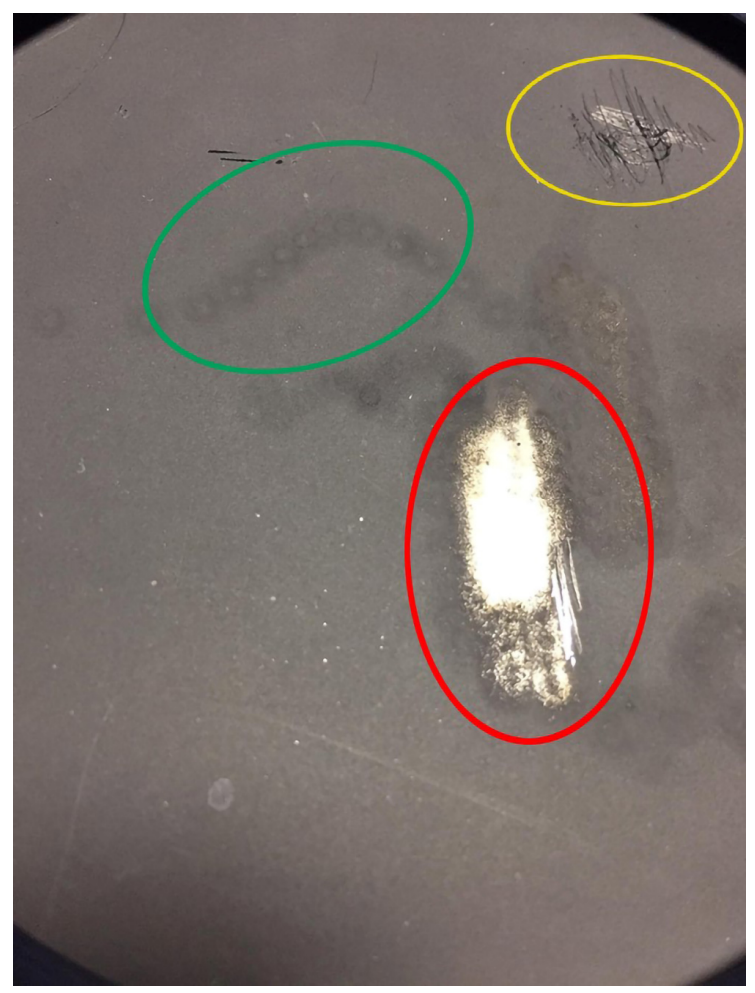

Fig. 5. Sections of the coating damage areas on the sensor. The green circle denotes the slightly damaged, the yellow circle represents the moderately damaged and the red circle outline the high damaged.

Slightly Damaged (SD), Moderately Damaged (MD), High Damaged (HD) coating performance of the sensor results were noted with loss percentages that is shown in Table 2. 
Table 2. Slightly Damaged (SD), Moderately Damaged (MD), High Damaged (HD) coating performance of the sensor

\begin{tabular}{|c|c|c|c|c|c|}
\hline $\begin{array}{c}\text { SD Energy } \\
\text { (mJ) }\end{array}$ & $\begin{array}{c}\text { SD Loss } \\
\mathbf{( \% )}\end{array}$ & $\begin{array}{c}\text { MD } \\
\text { Energy } \\
\text { (mJ) }\end{array}$ & $\begin{array}{c}\text { MD Loss } \\
\mathbf{( \% )}\end{array}$ & $\begin{array}{c}\text { HD Energy } \\
\text { (mJ) }\end{array}$ & $\begin{array}{c}\text { HD Loss } \\
\mathbf{( \% )}\end{array}$ \\
\hline 69.9 & 1.96 & 65.2 & 8.5 & 58.4 & 18 \\
\hline 59.9 & 1.8 & 56.7 & 7 & 51.1 & 16.2 \\
\hline 49.2 & 1.5 & 47.4 & 5 & 42.6 & 14.7 \\
\hline 39.5 & 1.3 & 38.5 & 4 & 34.7 & 13.4 \\
\hline 33.1 & 1.19 & 32.4 & 3.2 & 29.5 & 12.1 \\
\hline
\end{tabular}

Beam expander was used in all measurements taken from damaged and undamaged surfaces. Because, when taking these measurements, if the diameter of the laser beam is smaller than the damaged surface, measurements taken from highly damaged surfaces can cause full reflection.

\subsection{Uncertainty contribution}

Significant uncertainty contributions that may affect the measurement results can be on depends ambient temperature, the geometry of the laser on the detector, the absorption ability of the damaged coating material and the method of measurement. First of all, under the effects of temperature, test sensor is calibrated at $20^{\circ} \mathrm{C}$ and this sensor is used at $30{ }^{\circ} \mathrm{C}$. This $10^{\circ} \mathrm{C}$ temperature difference causes totally 2 percent energy deviation when considering 0.2 percent energy deviation over 1 Celsius degree. Secondly, the geometry of the detected laser within the aperture of the sensor will affect the measurement results. After the laser beam is expanded, measurements are taken as if they were theoretically perpendicular to the sensor. However, in practice it is very difficult to observe. For this reason, a standard deviation must be calculated by placing the laser beam at different angles on the sensor. After that, the losses must be calculated by taking measurements over the damaged coatings on the sensor and an appropriate coefficient must be determined for these calculations. Finally, the contribution of optics such as beam splitter, beam expander and polarizer used in measurements methods and the accuracy of the source must be added. 


\section{Discussions}

In summary, the aim of this study is to show how the damaged surfaces on the coatings of the detectors used in laser energy measurements affect the measurements results. With the help of the beam expander, the losses of the energy values taken from our damaged black coating sensor are given as a percentage that is shown in Table 3.

Table 3. Summary of the loss values for damaged coating performance of the sensor

\begin{tabular}{|c|c|}
\hline $\begin{array}{c}\text { Damaged } \\
\text { Coating } \\
\text { Performance }\end{array}$ & $\begin{array}{c}\text { Damaged Surface Lost } \\
\text { Performance }\end{array}$ \\
\hline SD & $1 \%-2 \%$ \\
\hline MD & $3 \%-9 \%$ \\
\hline HD & $12 \%-20 \%$ \\
\hline
\end{tabular}

Each detector user must map the faulty surfaces of their sensor by classifying the defective surfaces. All the results found are based only on the damaged surfaces of our detector.

For each specific measurement an uncertainty budget shall be created by considering these main items which contribute uncertainty. In the light of all above studies and results it has been determined that black coating on the energy sensors causing deviation between $\% 1$ and \%20 when considering damage levels. Regarding standards Test Uncertainty Ratio (TUR) of calibration providers should be 4 as a minimum requirement [5]. So this study also showed that damage level of sensor coating can decrease TUR value under minimum requirement for calibration providers since this deviation should be added to uncertainty budget directly. Further study may cover uncertainty approaches of sensor measurements in detailed.

T.O.K acknowledges the support of Roketsan Missiles Industries Inc.

\section{References}

1. W.R. Blevin, J. Geist, Influence of black coatings on pyroelectric detectors, Applied Optics 13, 5, 1171-1178, (1974)

2. R.W. Whatmore, Pyroelectric devices and materials, Reports Progress Phys. 49, 1335, (1986)

3. D. Lingam, A.R. Parikh, J. Huang, A. Jain, M.M. Jolandan, Nono/microscale pyroelectric energy harvesting : challanges and opportunities, International Journal of Smart and Nano Materials 4.4, 229-245, (2013)

4. D.B. Betts, F.J.J. Clarke, L.J. Cox, J.A. Larkin, Infrared reflection properties of five types of balck coating for radiometric detectors, Journal of Physics E: Scientific Instruments 18, 8, 689, (1985)

5. ASME B89.1.12-2001, Micrometers, American Society of Mechanical Engineers, (2001) 